\title{
The ethanolamide metabolite of DHA, docosahexaenoylethanolamine, shows immunomodulating effects in mouse peritoneal and RAW264.7 macrophages: evidence for a new link between fish oil and inflammation
}

\author{
Jocelijn Meijerink ${ }^{1 *}$, Pierluigi Plastina ${ }^{1}$, Jean-Paul Vincken ${ }^{2}$, Mieke Poland $^{1}$, Mohamed Attya ${ }^{1}$, \\ Michiel Balvers ${ }^{1,3}$, Harry Gruppen ${ }^{2}$, Bartolo Gabriele ${ }^{4}$ and Renger F. Witkamp ${ }^{1,3}$ \\ ${ }^{1}$ Division of Human Nutrition, Wageningen University, PO Box 8129, 6700 EV, Wageningen, The Netherlands \\ ${ }^{2}$ Laboratory of Food Chemistry, Wageningen University, PO Box 8129, 6700 EV, Wageningen, The Netherlands \\ ${ }^{3}$ TNO Quality of Life, PO Box 360, 3700 AJ Zeist, The Netherlands \\ ${ }^{4}$ Dipartimento di Scienze Farmaceutiche, Università della Calabria, 87036, Arcavacata di Rende, Cosenza, Italy
}

(Received 30 July 2010 - Revised 18 November 2010 - Accepted 3 December 2010 - First published online 4 February 2011)

\begin{abstract}
Several mechanisms have been proposed for the positive health effects associated with dietary consumption of long-chain $n$ - 3 PUFA ( $n$-3 LC-PUFA) including DHA (22:6n-3) and EPA (20:5n-3). After dietary intake, LC-PUFA are incorporated into membranes and can be converted to their corresponding $N$-acylethanolamines (NAE). However, little is known on the biological role of these metabolites. In the present study, we tested a series of unsaturated NAE on the lipopolysaccharide (LPS)-induced NO production in RAW264.7 macrophages. Among the compounds tested, docosahexaenoylethanolamine (DHEA), the ethanolamide of DHA, was found to be the most potent inhibitor, inducing a dose-dependent inhibition of NO release. Immune-modulating properties of DHEA were further studied in the same cell line, demonstrating that DHEA significantly suppressed the production of monocyte chemotactic protein-1 (MCP-1), a cytokine playing a pivotal role in chronic inflammation. In LPS-stimulated mouse peritoneal macrophages, DHEA also reduced MCP-1 and NO production. Furthermore, inhibition was also found to take place at a transcriptional level, as gene expression of MCP-1 and inducible NO synthase was inhibited by DHEA. To summarise, in the present study, we showed that DHEA, a DHA-derived NAE metabolite, modulates inflammation by reducing MCP-1 and NO production and expression. These results provide new leads in molecular mechanisms by which DHA can modulate inflammatory processes.
\end{abstract}

Key words: DHA: Docosahexaenoylethanolamine: Inflammation: Nitric oxide: Monocyte chemotactic protein-1: Macrophages

Fatty amides formed out of long-chain fatty acids and amino acids or ethanolamine widely occurr in nature. Increasing evidence suggests that many of these compounds are important signalling molecules in plants and animals, mediating a wide range of biological effects ${ }^{(1-3)}$. The most studied fatty amide so far is anandamide (arachidonoylethanolamine, AEA), which is one of the prototypical endogenous mediators of the endocannabinoid system ${ }^{(4,5)}$. Other fatty amides including $\mathrm{N}$-palmitoylethanolamine and $\mathrm{N}$-oleoylethanolamine have attracted great attention as lipid mediators involved in the anti-inflammatory processes ${ }^{(6,7)}$ and in the regulation of food intake $^{(8,9)}$. Also, $N$-stearoylethanolamine has been reported to have both anorexic ${ }^{(10)}$ and anti-inflammatory properties ${ }^{(11)}$. Based on the ubiquitous presence of enzyme systems that are able to synthesise amides, the hypothesis has been raised that any available long-chain fatty acid can be amidated with any amino acid or related compound ${ }^{(5)}$. Indeed, the number of amides detected in tissues is rapidly increasing ${ }^{(5,12)}$. Probably several of these 'novel' fatty amides might possess bioactivity.

For several fatty amides, it has been demonstrated that they can be rapidly synthesised from their fatty acid precursors into membranes, released on demand and quickly broken down ${ }^{(5,13)}$. Therefore, the local availability of a specific type of fatty acid precursor may determine product formation and hence bioactivity. This suggests a link with dietary intake, since it is well known that incorporation of fatty acids into membranes can be modulated by their proportional abundance in the diet. From a dietary perspective, $n-3$ long-chain PUFA ( $n$-3 LC-PUFA) such as DHA (22:6n-3) and EPA $(20: 5 n-3)$ are of great interest because of their potential

\footnotetext{
Abbreviations: AEA, arachidonoylethanolamine; BCA, bicinchoninic acid; DEA, docosatetraenoylethanolamine; DHEA, docosahexaenoylethanolamine; EPEA, eicosapentaenoylethanolamine; iNOS, inducible NO synthase; LPS, lipopolysaccharide; MCP-1, monocyte chemotactic protein-1; $n$-3 LC-PUFA, n-3 long-chain PUFA; NAE, $N$-acylethanolamine; RPMI-1640, Roswell Park Memorial Institute 1640; ZTT, tetrazolium salt.
} 
health-related benefits including a reduction in inflammatory processes and risks for CVD, type 2 diabetes and, possibly, certain neurological disorders ${ }^{(14-18)}$. It is well known that these compounds present in high concentrations in fatty fish are incorporated into membranes ${ }^{(15)}$ at the expense of that of arachidonic acid and other fatty acids. Although many mechanisms have been proposed, hardly anything is known about the possibility that conversion of $n-3$ LC-PUFA to fatty amides, thereby acting as bioactive lipid messengers, plays a role in these health-inducing effects. Evidence for the formation of $\mathrm{N}$-acylethanolamines (NAE) from EPA (eicosapentaenoylethanolamine (EPEA)) and DHA (docosahexaenoylethanolamine (DHEA)) in vivo has been published ${ }^{(19-22)}$. Furthermore, a few publications reporting NAE formation reveal a direct link between dietary intake of $n$-3 LC-PUFA and elevated EPEA and DHEA concentrations in animal tissues $^{(19,20)}$. Recently, we have demonstrated the formation of DHEA and EPEA by 3T3 adipocytes from their precursor fatty acids ${ }^{(23)}$. We have also found that DHEA was abundantly present in the human plasma of overnight fasted volunteers ${ }^{(23)}$. These observations raised further questions concerning the biological functions of DHEA, in particular, in relation to its immune-modulating effects.

First, a series of chemically closely related NAE, differing in the chain length and the number of double bonds, was evaluated for their ability to inhibit NO production by lipopolysaccharide (LPS)-stimulated RAW264.7 macrophages. NO, produced by inducible NO synthase (iNOS), is a late mediator induced during conditions of acute and chronic inflammation. Based on the results obtained, we further studied DHEA, demonstrating its action on monocyte chemotactic protein-1 (MCP-1), in vitro as well as in mouse peritoneal macrophages. Additionally, we studied whether DHEA-mediated effects are exerted at the gene expression level. Overall, the obtained results provide evidence for a novel molecular pathway mediating 'fish oil'/DHA-associated anti-inflammatory effects.

\section{Methods}

\section{Chemicals}

Oleoylethanolamine, DEA and AEA were purchased from Tocris (Bristol, UK). Novozym ${ }^{\circledR} 435$ was from Novozymes (A/S Bagsvaerd, Denmark). Dulbecco's modified Eagle's medium, Roswell Park Memorial Institute 1640 (RPMI-1640), fetal bovine serum, streptomycin and penicillin were acquired from Lonza (Verviers SPRL, Belgium). LPS was obtained from Sigma-Aldrich (Schnelldorf, Germany). Griess reagents and nitrite standard were purchased from Cayman Chemical (Ann Arbor, MI, USA). ELISA was performed using R\&D Systems kits (Abingdon, UK). Bicinchoninic acid (BCA) protein assay kit was acquired from Pierce (Rockford, IL, USA), and Brewer thioglycollate was from BD Sciences (Franklin Lakes, NJ, USA).

\section{$\mathrm{N}$-acylethanolamine synthesis}

NAE other than DEA, oleoylethanolamine and AEA (see Table 1 for the compounds tested) were prepared from ethanolamine
Table 1. Names of a series of $N$-acylethanolamines tested for their ability to inhibit nitric oxide production

\begin{tabular}{|c|c|c|}
\hline & $\mathrm{R}$ & Acyl chain \\
\hline Oleoylethanolamine & Oleic acid & $18: 1 n-9$ \\
\hline Linoleoylethanolamine & Linoleic acid & $18: 2 n-6$ \\
\hline Conjugated linoleoylethanolamine & $\begin{array}{l}\text { Conjugated } \\
\text { linoleic acid }\end{array}$ & $18: 2$ \\
\hline Linolenoylethanolamine & Linolenic acid & $18: 3 n-3$ \\
\hline Arachidonoylethanolamine & Arachidonic acid & $20: 4 n-6$ \\
\hline Eicosapentaenoylethanolamine & EPA & $20: 5 n-3$ \\
\hline Docosatetraenoylethanolamine & $\begin{array}{l}\text { Docosatetraenoic } \\
\text { acid }\end{array}$ & $22: 4 n-6$ \\
\hline Docosahexaenoylethanolamine & DHA & $22: 6 n-3$ \\
\hline
\end{tabular}

and their corresponding fatty acids using an enzymatic procedure as described earlier ${ }^{(24)}$. Briefly, the method is based on a direct condensation reaction between ethanolamine and the fatty acid (molar ratio $1: 1$ ), carried out at $40^{\circ} \mathrm{C}$ in hexane, using Novozym ${ }^{\circledR} 435$ (consisting of immobilised Candida antarctica Lipase B) as the catalyst. Incubation times ranged between 8 and $15 \mathrm{~h}$, and product yields obtained were in the range of 80 and $88 \%$. Authenticity of the products was verified by electrospray ionization-MS, ${ }^{1} \mathrm{H}$ NMR, ${ }^{13} \mathrm{C}$ NMR and Fourier transform infrared ${ }^{(24)}$. Purity of each compound was more than $98 \%$ as determined by HPLC and NMR.

\section{Cell culture}

RAW264.7 macrophages (murine origin) were obtained from the American Type Culture Collection (Teddington, UK). RAW264.7 macrophages were grown and maintained in Dulbecco's modified Eagle's medium supplemented with $10 \%$ fetal bovine serum, streptomycin and penicillin at $37^{\circ} \mathrm{C}$ in a $5 \% \mathrm{CO}_{2}$ humidified air atmosphere. Effects of the test compounds on the viability of the RAW264.7 cells were assessed using an XTT Cell Proliferation Kit II (Roche Applied Science, Almere, The Netherlands) according to the manufacturer's instructions. Briefly, cells were first incubated with the test compound(s). Thereafter, the tetrazolium salt (ZTT) assay was performed, where the cells' ability to metabolise XTT to formazan was a measure for cell viability. Conditions were considered toxic if metabolic activity to form formazan was decreased by $>20 \%$. As a negative control, Triton X100 was added to the cells, yielding total cell lysis. For further details on XTT assays, see Lupinacci et al. ${ }^{(25)}$.

\section{Peritoneal macrophages}

Male $\mathrm{C} 57 \mathrm{Bl} / 6$ mice were injected intraperitoneally with a $4 \%$ Brewer thioglycollate solution. After $3 \mathrm{~d}$, mice were killed, and cells in the peritoneal cavity were collected by peritoneal lavage, using RPMI-1640 medium containing penicillin/ streptomycin. Thereafter, peritoneal macrophages were collected by centrifugation for $5 \mathrm{~min}$ at $1250 \mathrm{rpm}$ and $4^{\circ} \mathrm{C}$, and pellets were subsequently treated with erythrocyte lysis 


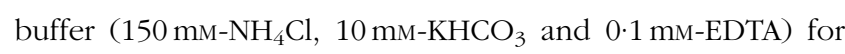
5-10 min on ice. Peritoneal macrophages were collected by centrifugation, and pellets were dissolved in RPMI-1640 containing $10 \%$ heat-inactivated fetal bovine serum and penicillin/streptomycin. Cells were counted and plated in a density of approximately 750000 cells/ml. After 2 h, adhering macrophages were washed with PBS and cultured for $72 \mathrm{~h}$ in RPMI-1640 medium (containing 10\% fetal bovine serum-heat-inactivated and penicillin/streptomycin) before stimulation.

For these experiments, the institutional and national guidelines for the care and use of animals were followed, and experiments were approved by the Local Committee for Care and Use of Laboratory Animals at Wageningen University.

\section{Effects of $\mathrm{N}$-acylethanolamines on nitric oxide release,} monocyte chemotactic protein-1 production and inducible nitric oxide synthase and monocyte chemotactic protein-1 gene expression

RAW264.7 macrophages $\left(2.5 \times 10^{5}\right.$ cells/ml) were seeded into ninety-six-well cell culture plates for nitrite, ELISA and cell viability measurements, or into six-well plates $\left(5 \times 10^{5}\right.$ cells $\left./ \mathrm{ml}\right)$ for the analysis of mRNA expression, and incubated overnight. Peritoneal macrophages were isolated and seeded as described earlier. Adherent cells were pre-incubated for $30 \mathrm{~min}$ with the test compound or vehicle in duplicate, after which LPS, $1 \mu \mathrm{g} / \mathrm{ml}$ for RAW264.7, was added in combination with the same test compound(s). For peritoneal macrophages, an optimal dose of $0 \cdot 1 \mu \mathrm{g}$ of LPS stimulation was assessed for the experimental conditions used. Therefore, peritoneal macrophages were activated with LPS $(0 \cdot 1 \mu \mathrm{g} / \mathrm{ml})$ to measure MCP-1 release. However, because of nitrite detection limits, LPS $(1 \mu \mathrm{g} / \mathrm{ml})$ was used to measure NO production. Ethanol was used (final solvent concentration never exceeded $0 \cdot 1 \%$, $\mathrm{v} / \mathrm{v})$ as the solvent. Incubation times were selected based on the specific properties of different inflammatory mediators. After incubating for 24 or $48 \mathrm{~h}$, nitrite accumulated in the culture medium was measured as an indicator of $\mathrm{NO}$ production using the Griess method ${ }^{(26)}$. Briefly, $100 \mu l$ of the cell culture medium were mixed with $100 \mu \mathrm{l}$ of Griess reagents and incubated at room temperature for $10 \mathrm{~min}$. Absorbance was measured at $540 \mathrm{~nm}$ using an ELISA plate reader. Following 4 or $16 \mathrm{~h}$ incubation, MCP-1 levels were assessed by using ELISA.

\section{RNA purification and quantitative reverse transcription real-time $P C R$}

Total RNA was extracted using TRIzol ${ }^{\mathrm{R}}$ (Invitrogen, Breda, The Netherlands). RNA ( $1 \mu \mathrm{g} / \mathrm{sample})$ was reverse transcribed to give complementary DNA using the reverse transcription system from Promega (Leiden, The Netherlands). Complementary DNA was amplified by PCR using platinum Taq DNA polymerase (Invitrogen) and SYBR green (Molecular Probes, Leiden, The Netherlands) on an iCycler apparatus (Bio-Rad, Veenendaal, The Netherlands). The following primer pairs were used for amplification of iNOS:
5'-GTT-CTC-AGC-CCA-ACA-ATA-CAA-GA-3' (forward) and 5'-GTG-GAC-GGG-TCG-ATG-TCA-C-3' (reverse); MCP-1: $5^{\prime}$ CCC-AAT-GAG-TAG-GCT-GGA-GA- $3^{\prime}$ (forward) and $5^{\prime}$-TCTGGA-CCC-ATT-CCT-TCT-TG-3' (reverse). Samples were analysed in duplicate, and mRNA expression levels of the different genes were normalised to RPS27A2. Primer pairs for RPS27A2 were $5^{\prime}$-GGT-TGA-ACC-CTC-GGA-CAC-TA-3' (forward) and 5'-GCC-ATC-TTC-CAG-CTG-CTT-AC-3' (reverse).

\section{Statistical analysis}

All experiments in RAW264.7 macrophages were performed in duplicate, and those performed in the peritoneal macrophages were in triplicate in at least three independent experiments. Data from all experiments are expressed as percentage of the LPS-treated controls (set at 100\%). Data are presented as means and standard errors of the mean (see legends of the figures). Statistical differences between treatments and controls were evaluated by two-way ANOVA followed by Bonferroni's post hoc test. A $P$ value $<0.05$ was considered as statistically significant. $P$ values were assigned at three different levels, namely $P<0.05,<0.01$ and $<0.001$.

\section{Results}

Effect of $\mathrm{N}$-acylethanolamines with different chain lengths and number of double bonds on lipopolysaccharideinduced nitric oxide production

Fatty acid-derived NAE, chemically closely related to DHEA, were compared for their immune-modulating properties. Therefore, a series of NAE differing in chain length (from $\mathrm{C} 18$ to $\mathrm{C} 22$ ) and number of double bonds ( $1 n$ to $6 n$ ) were tested for their abilities to inhibit LPS-induced nitric oxide production in RAW264.7 macrophages. NO is a late inflammatory marker and can be determined in LPS-stimulated macrophages from $24 \mathrm{~h}$ onwards, with levels still increasing up to $48 \mathrm{~h}$ and possibly later. The effect on NO release was determined at two time points ( 24 and $48 \mathrm{~h}$ ), with concentration dependencies assessed at $48 \mathrm{~h}$. Table 1 shows the molecular structures of the NAE tested. Oleoylethanolamine and other compounds having a length of eighteen carbons did not inhibit NO production either at $24 \mathrm{~h}$ (Fig. 1) or at $48 \mathrm{~h}$ (Fig. 2). Among the C20 compounds, AEA did not affect NO levels at $t=24 \mathrm{~h}$ but inhibited NO release at $t=48 \mathrm{~h}$ in a slight and significant way. In contrast, EPEA $(20: 5)$ and DEA (22:4) significantly decreased NO release at $t=24 \mathrm{~h}$ (Fig. 1) and reduced NO production in a significant way at concentrations of 1 and $10 \mu \mathrm{M}$ at $t=48 \mathrm{~h}$ (Fig. 2). At a concentration of $10 \mu \mathrm{M}$, DHEA (22:6) significantly suppressed NO production up to $40 \%$ at $t=24 \mathrm{~h}$ (Fig. 1) and in a concentration-dependent way at $t=48 \mathrm{~h}$ (Fig. 2). The highest concentration of $10 \mu \mathrm{M}$ gave a more than $60 \%$ reduction at $t=48$, while $1 \mu \mathrm{M}$ decreased NO production by $20 \%$. Generally, the potency of NAE to reduce NO release was enhanced with their increasing chain length and number of double bonds. The precursor of DHEA, DHA, produced only little suppression of NO production. 


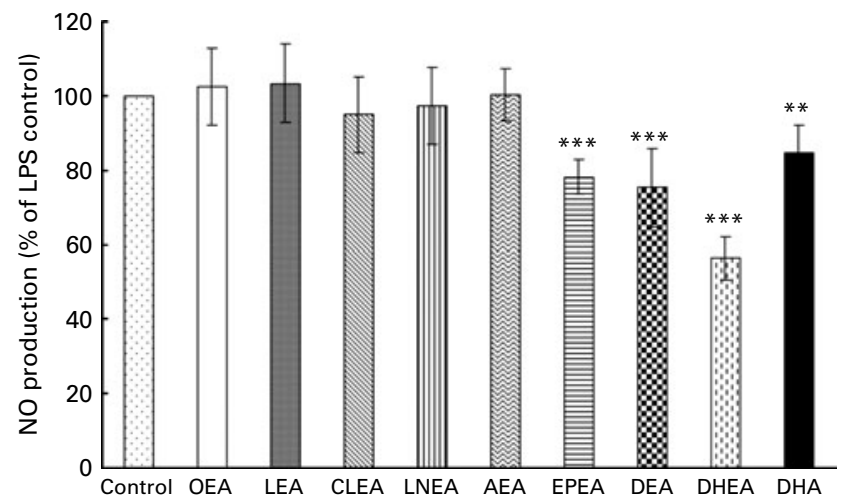

Fig. 1. Efficacy of a series of $N$-acylethanolamines to inhibit lipopolysaccharide (LPS)-induced nitric oxide release in RAW264.7 macrophages at $24 \mathrm{~h}$. Efficacies were compared with nitric oxide-reducing effects of the precursor DHA. All compounds were tested at a dose of $10 \mu \mathrm{M}$. RAW264.7 macrophages were seeded at a density of $250000 \mathrm{cells} / \mathrm{ml}$ and pre-incubated for $30 \mathrm{~min}$ with the respective ligands preceding a $24 \mathrm{~h} \mathrm{LPS}(1 \mu \mathrm{g} / \mathrm{ml})$ stimulation, in the presence of the particular ligand. Protein content of each well was determined by a bicinchoninic acid assay. Data are expressed as percentage, where LPS stimulation (containing vehicle) was set at $100 \%$. Average absolute value for nitrite production with LPS stimulation (control) after $24 \mathrm{~h}$ was approximately $16 \mu \mathrm{m}$. Values are means of four separate experiments (each done in duplicate), with standard errors of the mean represented by vertical bars. Mean values were significantly different from $100 \%$ : ${ }^{* *} P<0.01$, ${ }^{* \star *} P<0.001$. OEA, oleoylethanolamine; LEA, linoleoylethanolamine; CLEA, conjugated linoleoylethanolamine; LNEA, linolenoylethanolamine; AEA, arachidonoylethanolamine; EPEA, eicosapentaenoylethanolamine; DEA, docosatetraenoylethanolamine; DHEA, docosahexaenoylethanolamine.

Only the highest concentration tested elicited a significant NO decrease both after 24 and $48 \mathrm{~h}$.

Cell viability was verified using an XTT cell proliferation kit assay. Neither LPS alone nor LPS in combination with the highest concentration $(10 \mu \mathrm{M})$ of different NAE affected the viability of cells after $24 \mathrm{~h}$. At $48 \mathrm{~h}$, cell viability slightly dropped to a range of $83-87 \%$ but only in the LPS-treated cells. Cell viability was not further decreased by adding the tested components, indicating that the observed effect was caused by LPS. Conditions are regarded toxic if cell viability lowers more than $20 \%$. As all the tested compounds were within the viability range of $83-100 \%$, experimental conditions were considered non-toxic.

\section{Docosahexaenoylethanolamine elicits dose-dependent suppression of monocyte chemotactic protein-1}

Out of the NAE series, DHEA turned out to be the most effective component suppressing LPS-induced NO release. These results prompted us to further focus on DHEA and to investigate its effects on MCP-1, a cytokine playing a pivotal role in inflammatory processes. In contrast to the late inflammatory marker nitric oxide, MCP-1 is a relatively early marker of the LPS-induced inflammatory cascade. MCP-1 production induced by LPS stimulation in RAW264.7 macrophages is already observed at $4 \mathrm{~h}$, with levels further increasing to reach maximum levels at $16 \mathrm{~h}$ to stay high until later time points ( $\mathrm{J}$ Meijerink, unpublished results). In order to assess whether DHEA reduces cytokine production also at earlier time points during the inflammatory cascade, MCP-1 levels were determined both at $4 \mathrm{~h}$ and at $16 \mathrm{~h}$ when MCP-1 has reached maximum levels. Pre-incubation with DHEA dosedependently reduced LPS-induced MCP-1 production in RAW264.7 macrophages both at $4 \mathrm{~h}$ (Fig. 3(a)) and at $16 \mathrm{~h}$ (Fig. 3(b)). At $16 \mathrm{~h}$, a significant inhibition of $53 \%(P<0.001)$ was elicited by $10 \mu \mathrm{m}-\mathrm{DHEA}$, while $100 \mathrm{~nm}$ still significantly evoked a reduction of $18 \%(P<0.05)$. The dose-response curve at $4 \mathrm{~h}$ shows an almost identical curve compared with the $16 \mathrm{~h}$ time point, with the only difference being the lack of statistical significance for the MCP-1 reduction elicited by $100 \mathrm{~nm}$-DHEA at $4 \mathrm{~h}$. The lowest dose for DHEA-induced reduction in $\mathrm{MCP}-1$, observed at $16 \mathrm{~h}$, was approximately ten times lower than the dose needed to reduce NO levels.

\section{Anti-inflammatory effects of docosahexaenoylethanolamine on mouse peritoneal macrophages}

To study whether DHEA exerts similar anti-inflammatory effects under ex vivo conditions as in vitro, thioglycollateelicited peritoneal macrophages were exposed to different concentrations of DHEA and subsequently activated by LPS. Pre-incubation with DHEA resulted in diminished levels of both NO (Fig. 4(a)) and MCP-1 (Fig. 4(b)). A dose-dependent effect was found for both markers. Due to the limited lifespan of the primary peritoneal macrophages, NO measurements were performed at $24 \mathrm{~h}$ rather than at $48 \mathrm{~h}$ when maximal levels of production were reached. At the $24 \mathrm{~h}$ time point, $10 \mu \mathrm{M}$-DHEA significantly inhibited NO release up to $47 \%$ (Fig. 4(a)). Effects are comparable with those exerted in RAW264.7 cells (Fig. 1). Also, a DHEA-elicited concentrationdependent effect was found for MCP-1 at $16 \mathrm{~h}$, with the lowest dose of $1 \mu \mathrm{m}$ being still significant. Effects were less pronounced as those assessed in RAW264.7 macrophages. Cell viability of peritoneal macrophages during experimental conditions was determined using an XTT assay and considered non-toxic.

\section{Docosahexaenoylethanolamine suppresses lipopolysaccharide-induced mRNA expression of inducible nitric oxide synthase and monocyte chemotactic protein-1}

In order to investigate whether $\mathrm{NO}$ and MCP-1 inhibition elicited by DHEA is regulated at the gene expression level, we used quantitative RT-PCR to determine iNOS (Fig. 5(a)) and MCP-1 (Fig. 5(b)) mRNA expression. A concentration of $10 \mu \mathrm{M}$-DHEA significantly suppressed LPS-induced iNOS mRNA gene expression at $24 \mathrm{~h}$ up to $52 \%$. A similar doseresponse relationship was found for MCP-1 gene expression, where the highest dose of DHEA tested resulted in a reduction of $51 \%$. Lowest doses eliciting significant reduction were considerably higher at the gene expression level than at the production level.

\section{Discussion}

Despite the high abundance of nutritionally important $n$-3 LC-PUFA in brain and other tissues ${ }^{(27-30)}$, remarkably little has been described so far on their conjugation with amines 

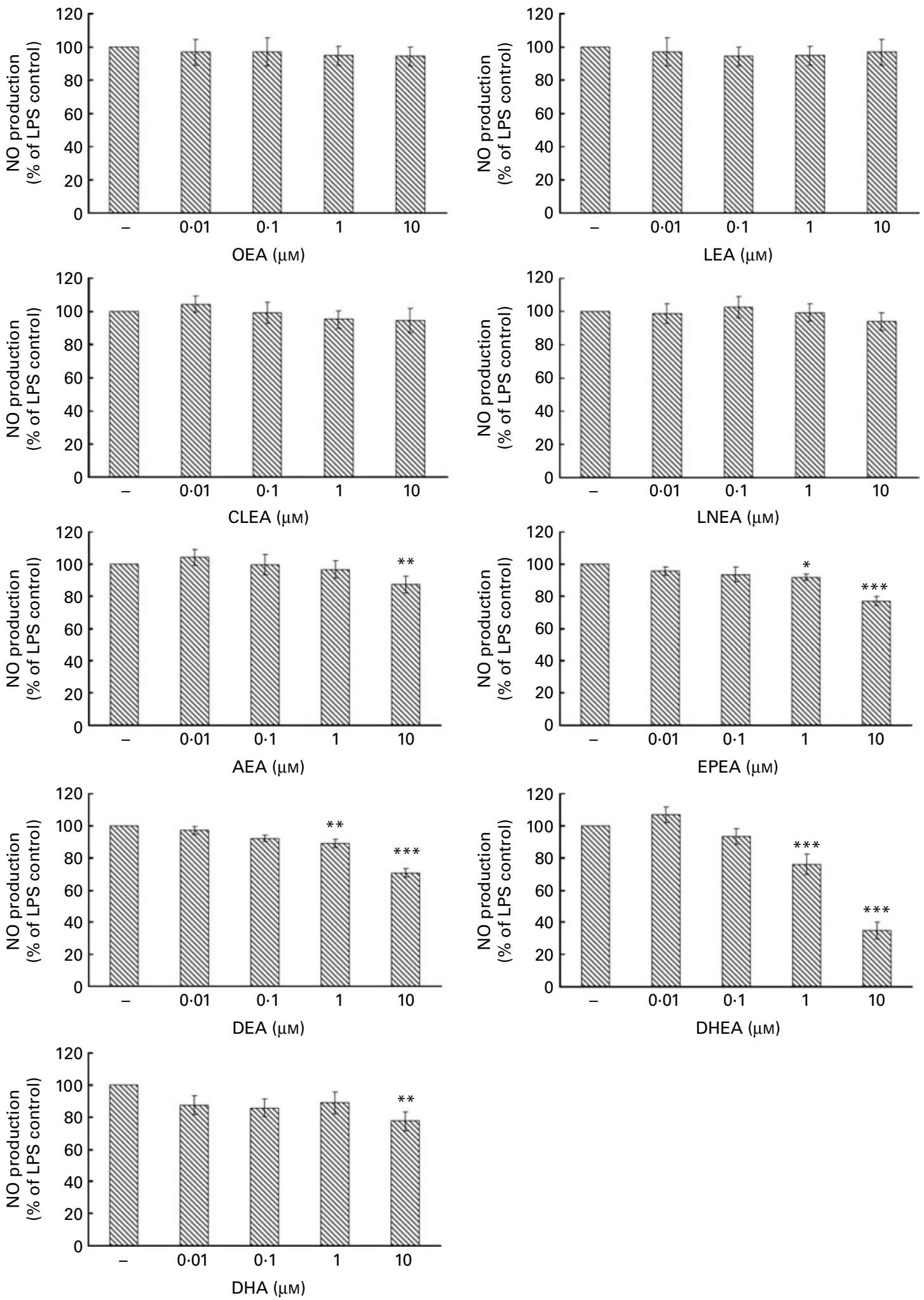

Fig. 2. Dose-response graphs of lipopolysaccharide (LPS)-induced nitric oxide reduction elicited by a series of $N$-acylethanolamines and DHA in RAW264.7 macrophages. Compounds were tested in a concentration range of 0.01-10 $\mu \mathrm{M}$. RAW264.7 macrophages were seeded at a density of $250000 \mathrm{cells} / \mathrm{ml}$ and preincubated for $30 \mathrm{~min}$ with the respective ligands before a $48 \mathrm{~h} \mathrm{LPS}(1 \mu \mathrm{g} / \mathrm{ml})$ stimulation, in the presence of the particular ligand. The protein content of each well was determined by a bicinchoninic acid assay. Data are expressed as percentage, where LPS stimulation (containing vehicle) was set at $100 \%$. Average absolute value for nitrite production with LPS stimulation (control) after $48 \mathrm{~h}$ was approximately $45 \mu \mathrm{M}$. Values are means of four separate experiments (each done in duplicate), with standard errors of the mean represented by vertical bars. Mean values were significantly different from the control: ${ }^{\star} P<0.05,{ }^{* \star} P<0.01,{ }^{\star \star \star *} P<0.001$. OEA, oleoylethanolamine; LEA, linoleoylethanolamine; CLEA, conjugated linoleoylethanolamine; LNEA, linolenoylethanolamine; AEA, arachidonoylethanolamine; EPEA, eicosapentaenoylethanolamine; DEA, docosatetraenoylethanolamine; DHEA, docosahexaenoylethanolamine. 
(a)

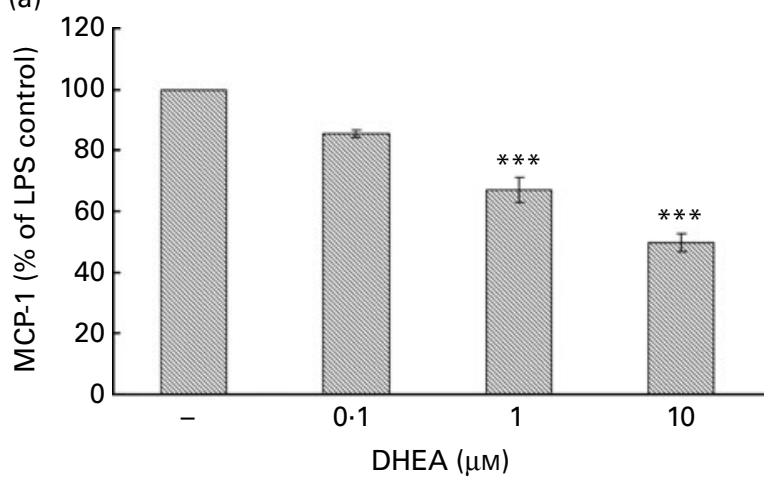

(b)

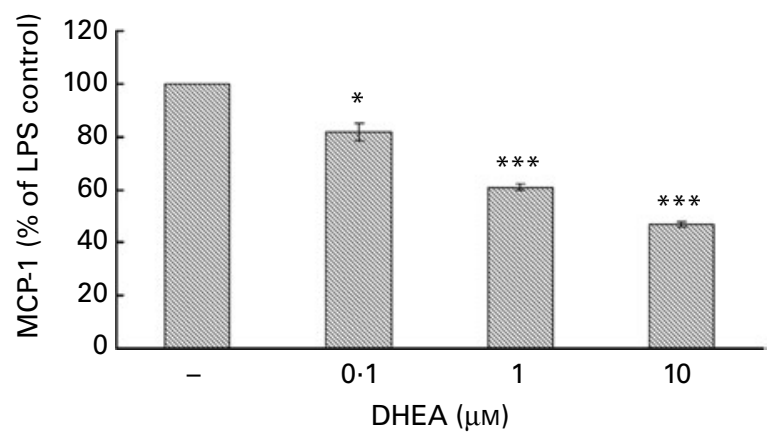

Fig. 3. Effect of docosahexaenoylethanolamine (DHEA) on monocyte chemotactic protein-1 (MCP-1) cytokine production in RAW264.7 macrophages at different time points. RAW264.7 cells were seeded at a density of 250000 cells $/ \mathrm{ml}$ and pre-incubated with a concentration series of DHEA for $30 \mathrm{~min}$ before (a) a $4 \mathrm{~h}$ lipopolysaccharide (LPS, $1 \mu \mathrm{g} / \mathrm{ml}$ ) stimulation in the presence of DHEA and (b) a $16 \mathrm{~h} \mathrm{LPS}(1 \mu \mathrm{g} / \mathrm{ml})$ stimulation in the presence of DHEA. The supernatant of the cells was analysed for MCP-1 production by ELISA. The protein content of each well was determined by a bicinchoninic acid assay. Values for MCP-1 production were corrected for the amount of protein for each well separately. Data are expressed as percentage, where LPS stimulation (containing vehicle) was set at $100 \%$. Values are means of three separate experiments (each done in duplicate), with standard errors of the mean represented by vertical bars. Mean values were significantly different from the control: ${ }^{*} P<0.05,{ }^{\star \star \star} P<0.001$.

into fatty amides. In addition, hardly anything is known about the potential biological significance of these amides. About 10 years ago, the presence of DHEA in bovine retina was demonstrated by Bisogno et al. ${ }^{(21)}$. A few years later, Berger et al. ${ }^{(20)}$ showed that brain levels of EPEA and DHEA were highly increased after feeding newborn piglets a milk formula rich in EPA and DHA. More recently, increased levels of DHEA and EPEA were demonstrated in the liver and jejunum of rats that had been fed a high-fish oil diet ${ }^{(19)}$. In a recently published study, we showed that DHA can be rapidly and efficiently converted to its ethanolamide, DHEA, by 3T3-L1 adipocytes $^{(23)}$. This is in accordance with the findings of Eckardt et al. ${ }^{(22)}$ who demonstrated the presence of DHEA among other NAE in the culture medium of human primary pre-adipocytes. In the present study, we investigated the potential biological implications of these findings and established that DHEA, a 'novel' bioactive member of the group of NAE, possesses immune-modulating properties in macrophages.
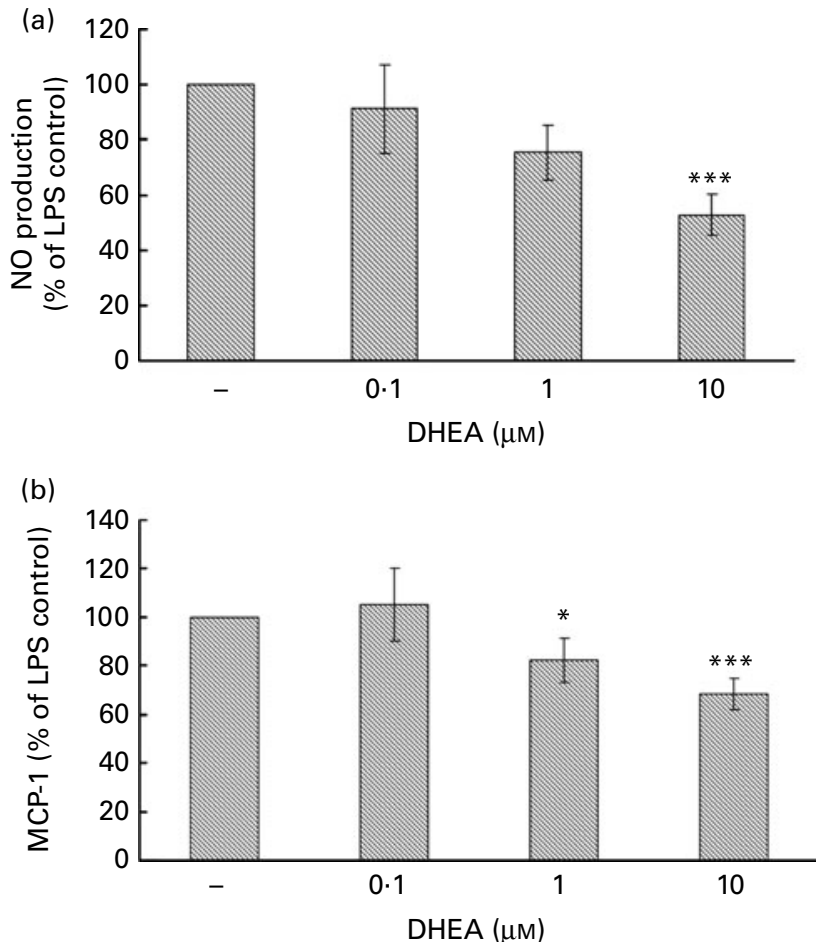

Fig. 4. Effect of docosahexaenoylethanolamine (DHEA) on monocyte chemotactic protein-1 (MCP-1) cytokine and nitric oxide production in peritoneal macrophages. Cells were seeded at a density of approximately 750000 cells $/ \mathrm{ml}$ and pre-incubated with a concentration series of DHEA $30 \mathrm{~min}$ before (a) a $24 \mathrm{~h}$ lipopolysaccharide (LPS, $1 \mu \mathrm{g} / \mathrm{ml}$ ) stimulation in the presence of DHEA for nitric oxide determination and (b) a $16 \mathrm{~h}$ LPS $(0.1 \mu \mathrm{g} / \mathrm{ml})$ stimulation in the presence of DHEA for MCP-1 determination. The supernatant of the cells was analysed for nitric oxide using the Griess assay and for MCP-1 by ELISA. The protein content of each well was measured by a bicinchoninic acid assay. Values for MCP-1 and nitric oxide production were corrected for the amount of protein for each well separately. Data are expressed as percentage, where LPS stimulation (containing vehicle) was set at $100 \%$. Data for nitric oxide represent means of cells isolated from five different mice (with each biological sample performed in duplicate or triplicate), except for the doses of $0.1 \mu \mathrm{M}$-DHEA that was performed in three mice, with standard errors of the mean represented by vertical bars. For MCP-1 analysis, four mice were used (with each biological sample performed in duplicate or triplicate). Mean values were significantly different from the control: ${ }^{\star} P<0.05$, ${ }^{\star \star *} P<0.001$

\section{Effects on nitric oxide release}

NO produced by iNOS has been shown to be a key inflammatory mediator in several diseases associated with the metabolic syndrome. Disruption of iNOS, by using knockout mice models, was found to diminish diet-induced atherosclerosis and improve insulin signalling and glucose tolerance in obese mice ${ }^{(31-33)}$. NO is a signalling molecule produced in the oxidative deamination of L-arginine catalysed by a NOS During an inflammatory process, the inducible isoform of this enzyme (iNOS or NOS2), which is not expressed in non-pathological situations, is up-regulated ${ }^{(34,35)}$. This upregulation can be initiated by microbial products such as LPS, inflammatory cytokines or interferon- $\gamma$.

Of the NAE tested in the present study, we found that DHEA had the highest potency to inhibit NO formation in LPSstimulated RAW264.7 macrophages. For the C18 chain-length components, the number and position of double bonds did 


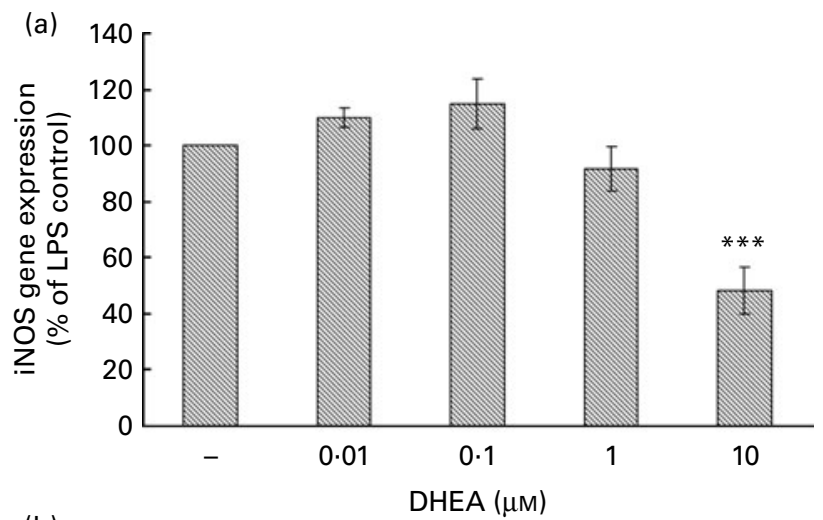

(b)

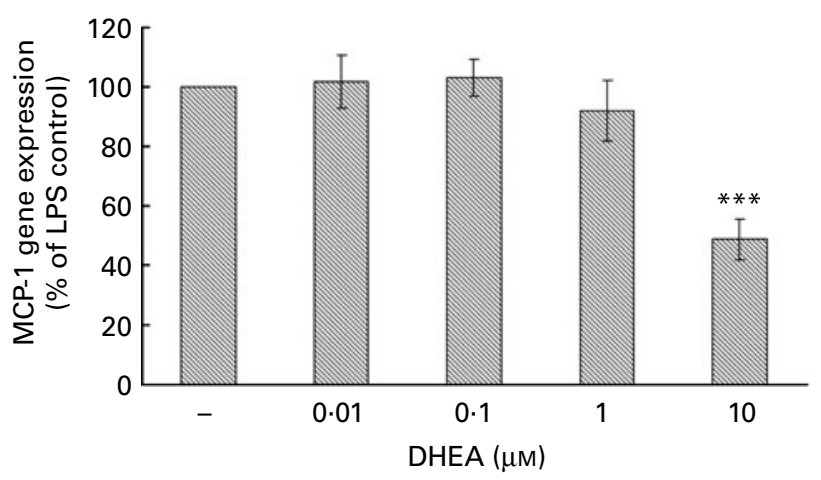

Fig. 5. Effect of docosahexaenoylethanolamine (DHEA) on lipopolysaccharide (LPS)-induced inducible nitric oxide synthase (iNOS) and monocyte chemotactic protein-1 (MCP-1) mRNA expression at 24h. RAW264.7 cells were seeded at a density of 500000 cells $/ \mathrm{ml}$ and pre-incubated with a concentration series of DHEA for $30 \mathrm{~min}$ before a $24 \mathrm{~h}$ LPS stimulation in the presence of DHEA. Total RNA was isolated and reverse transcribed to give complementary DNA before performing quantitative real-time PCR. Details are described in the Methods section. iNOS and MCP-1 fold increase normalised to RPS27A2 is expressed as percentage, where LPS stimulation (without DHEA) was set at $100 \%$. Values are means of three separate experiments performed in duplicate, with standard errors represented by vertical bars. ${ }^{\star * \star}$ Mean value was significantly different from that of the control $(P<0.001)$

not influence the activity of the fatty amides; none of them being effective in inhibiting NO release up to a concentration of $10 \mu \mathrm{M}$. Increasing the chain length demonstrated that AEA (20:4; anandamide), a well-known endocannabinoid, inhibited NO release at $t=48 \mathrm{~h}$ in a slight and significant way. Further increase in the number of double bonds $(20: 5)$ and/or chain length $(22: 4,22: 6)$ led to an increased capacity to inhibit nitric oxide production, with that of DHEA (22:6n-3) being detectable at a concentration of $0 \cdot 1 \mu \mathrm{m}$. Its precursor DHA only had a small effect in our assay. This might be in line with results reported by Weldon et al. ${ }^{(36)}$ who needed relatively high concentrations $(100 \mu \mathrm{M})$ of DHA and a pre-incubation period to achieve inhibition of the release of inflammatory mediators in LPS-stimulated THP-1 (human leukaemic monocyte cell line) macrophages. It also indicates that the effects of DHEA found are not due to its hydrolysis to DHA, which could occur via fatty acid amide hydrolase ${ }^{(37)}$. Control studies performed in our group using LC-MS to assess the stability of DHEA in the experimental set-up has shown that DHEA concentrations slightly declined with a $10 \%$ reduction at $2 \mathrm{~h}$ to reach an approximately $75 \%$ decrease after $48 \mathrm{~h}$ (M Balvers, unpublished results). This shows that doses of DHEA were in the active range $(>1 \mu \mathrm{M})$ during the entire experiment. Moreover, although NO has been measured at $48 \mathrm{~h}$, data represent DHEA-elicited effects of a late marker of the inflammatory cascade. It is, however, very likely that DHEA already exerts its effects early during the initiation of the cascade. Data for the $4 \mathrm{~h}$ time point support this theory, as DHEA significantly reduced levels of the relatively early marker MCP-1 at $4 \mathrm{~h}$. Taken together, all data indicate that the DHEA-elicited immunomodulatory effects can be explained from the activity of DHEA itself.

\section{Effects of docosahexaenoylethanolamine on monocyte chemotactic protein-1 protein and mRNA levels}

Similar to NO, MCP-1 has been shown to be a key inflammatory mediator in metabolism-related disorders. The proinflammatory chemokine MCP-1 functions as a leucocyte chemoattractor and is predominantly produced by macrophages or endothelial cells. Blocking expression of MCP-1 or its receptor under conditions leading to artherosclerosis in mice resulted in diminished formation of atherosclerotic lesions and lipid deposition in their aortas ${ }^{(38,39)}$. Furthermore, in obesity, a casual relationship for MCP-1 and macrophage infiltration in adipose tissue, insulin resistance and hepatic steatosis has been established $^{(40)}$, and only recently, another study has shown that high levels of circulating MCP-1 in the plasma are crucial for inducing insulin resistance in mice ${ }^{(41)}$.

We found that DHEA dose-dependently reduced MCP-1 production in both RAW264.7 and thioglycollate-elicited mouse peritoneal macrophages. The lowest dose at which DHEA inhibited MCP-1 release was at $100 \mathrm{~nm}$ at $16 \mathrm{~h}$ in RAW264.7 cells. Minimum doses eliciting significant reduction were a factor 100-1000 lower than those reported in the literature for anti-inflammatory effects mediated by its precursor DHA or for the $n-3$ PUFA, EPA ${ }^{(42,43)}$. Interestingly, Shi \& Pestka $^{(44)}$ reported that in peritoneal macrophages of mice, which were fed a DHA-enriched diet for several weeks, antiinflammatory pathways were induced, which were not found when naive peritoneal macrophages were directly exposed to DHA. The authors suggest that DHA-derived metabolites might be responsible for the immune-modulating effects in vivo rather than DHA itself.

Our data showed that DHEA elicited a suppression of both MCP-1 and iNOS mRNA. These results suggest that DHEAinduced inhibition is primarily regulated at the mRNA level. However, although anti-inflammatory effects at a dose of $10 \mu \mathrm{m}$ reflect inhibition as determined at the protein level, dose-dependent effects in mRNA expression were only slightly visible, with $1 \mu \mathrm{m}$ being the lowest dose showing a small non-significant reduction. Consequently, it cannot be ruled out that DHEA-elicited effects are regulated by different mechanisms, with lower doses regulated at the protein level, while suppressive effects at higher doses are regulated at the expression level. Alternatively, this discrepancy might also be caused by differences in relative sensitivity of the techniques used. 


\section{n-3 Long-chain PUFA and inflammation: alternative mechanisms of action}

It is well known that eating a diet rich in $n$-3 LC-PUFA, for example fatty fish, leads to a gradual incorporation of $n-3$ LC-PUFA in cellular membranes of different tissues at the expense of other fatty acids including arachidonic $\operatorname{acid}^{(29,45)}$. This has also been described for cells of the immune system, causing changes in eicosanoid production patterns and cellular functionality ${ }^{(14)}$. The fatty acid composition and positional distribution of dietary TAG have also consequences for the relative amounts of 2-acylglycerols formed. This may affect CB1/CB2 receptor binding, either directly (via $s n$-2-arachidonoylglycerol) or indirectly, for example via an 'entourage' effect. EPA and DHA also serve as precursors for resolvins, another class of inflammatory mediators ${ }^{(14,15,46)}$. Resolvins or other DHA eicosanoid products can form novel $N$-linked ethanolamines that may be biologically active ${ }^{(4,27)}$. The formation of amides from $n$-3 LC-PUFA, as shown in the present study, might represent a new branch on the tree of pathways involved in the complex regulation of inflammation. Endocannabinoid and eicosanoid pathways are known to share a number of common biotransformation pathways, regulatory enzymes and, possibly, receptors ${ }^{(47)}$. Local availability of precursors can play a role in the inflammatory balance, as has been suggested for $n-3 v, n-6$ in the $\operatorname{diet}^{(45,48)}$. However, it is also clear that there is not a direct stoichiometric relationship between dietary intake of precursors and production of inflammatory mediators. Instead, a well-regulated homeostatic equilibrium exists that fluctuates between rather narrow boundaries ${ }^{(18,45,46)}$. However, in the case of endocannabinoids, dietary studies have clearly shown that this equilibrium is subject to gradual changes. Although there are only few studies showing direct formation of NAE from $n$-3 LC-PUFA precursors, several other studies have shown that dietary intervention with $n-3$ fatty acids also indirectly affects the endocannabinoid balance. For example, Watanabe et al. ${ }^{(49)}$ have demonstrated that $n$-3 LCPUFA deficiency elevates and $n$-3 LC-PUFA administration reduces brain $s n$-2-arachidonoylglycerol levels in mice. Batetta et al. ${ }^{(50)}$ have recently shown that diets rich in $n-3$ LC-PUFA decreased AEA and $s n$-2-arachidonoylglycerol levels in the visceral fat of obese Zucker rats. In the study of Artmann et $a l .{ }^{(19)}$, decreased levels of all NAE measured were found in the livers of rats fed with n-3 LC-PUFA for 1 week, except for DHEA and EPEA. However, in the brain, these effects were not observed, which may be due to the short treatment period. Taken together, it appears that NAE patterns tend to follow the relative abundance of lipids in the diet. However, a few weeks of intake are needed to achieve significant changes. In addition to stimulating the formation of DHEA by increasing dietary supply, inhibition of its breakdown by fatty acid amide hydrolase could further elevate local concentrations $^{(37)}$. Some NAE, including arachidonoyl serotonin, are known to have fatty acid amide hydrolase-inhibiting properties $^{(51,52)}$. Hence, NAE that do not bind to CB1 or CB2 can still exert biological action in an indirect way.

\section{Is there a role for docosahexaenoylethanolamine as an} immune modulator?

In relation to the reported health effects of fish oil and $n$ - 3 LCPUFA, our findings might be of interest for several applications and areas. One such area is obesity/metabolic syndrome, where an inflammatory process plays a role ${ }^{(53-56)}$. A biochemical connection between fish oil intake and the pathology of obesity has recently been suggested by Batetta et al. ${ }^{(50)}$, showing that $n-3$ LC-PUFA supplementation inhibited the inflammatory process and ectopic fat deposition while reducing AEA in obese Zucker rats. Several studies have demonstrated the importance of $\mathrm{NO}$ in obesity and the metabolic syndrome ${ }^{(57-60)}$. Fish oil has also shown potential beneficial activity in inflammatory diseases of the intestinal tract $^{(61)}$, and high incorporation of DHEA and EPEA was found in gut tissue after a fish oil diet ${ }^{(19)}$. In conclusion, our data provide evidence for a novel mechanism by which DHA exerts immune-modulating effects, namely as a precursor of DHEA. Combined with findings from previous studies, our data provide new viewpoints on the relationship between dietary intake of DHA and local inflammatory processes. It is tempting to speculate that DHEA might possibly play a significant role in inflammatory processes in vivo, thereby functioning as an endogenous 'natural' ligand. Further studies should elucidate the relevance of this 'fish oil messenger' in vivo and the relationship to other inflammatory pathways in which DHA is involved.

\section{Acknowledgements}

The present study has been supported by research grants from Wageningen University (The Netherlands). P. P. received a grant for a postdoctoral fellowship from Fondazione Carical (Cosenza, Italy). In addition, TNO (Zeist, The Netherlands) has supported part of the study (Balance/VP9). J. M. and R. F. W. designed the study and wrote the manuscript. P. P. performed the experimental work and contributed to the design and preparation of the manuscript. J.-P. V. designed the study. H. G. and B. G. facilitated the experimental work, and M. P., M. A. and M. B. performed the experimental work. The authors declare that there is no conflict of interest.

\section{References}

1. Kilaru A, Blancaflor EB, Venables BJ, et al. (2007) The $\mathrm{N}$-acylethanolamine-mediated regulatory pathway in plants. Chem Biodivers 4, 1933-1955.

2. Okamoto Y, Wang J, Morishita J, et al. (2007) Biosynthetic pathways of the endocannabinoid anandamide. Chem Biodivers 4, 1842-1857.

3. Borrelli F \& Izzo AA (2009) Role of acylethanolamides in the gastrointestinal tract with special reference to food intake and energy balance. Best Prac Res Clin Endocrinol Metab 23, 33-49.

4. Alexander SPH \& Kendall DA (2007) The complications of promiscuity: endocannabinoid action and metabolism. Br J Pharmacol 152, 602-623.

5. Di Marzo V, Bisogno T \& De Petrocellis L (2007) Endocannabinoids and related compounds: walking back and forth 
between plant natural products and animal physiology. Chem Biol 14, 741-756.

6. Re G, Barbero R, Miolo A, et al. (2007) Palmitoylethanolamide, endocannabinoids and related cannabimimetic compounds in protection against tissue inflammation and pain: potential use in companion animals. Vet J 173, 21-30.

7. Hoareau L, Buyse M, Festy F, et al. (2009) Anti-inflammatory effect of palmitoylethanolamide on human adipocytes. Obesity 17, 431-438.

8. Thabuis C, Tissot-Favre D, Bezelgues JB, et al. (2008) Biological functions and metabolism of oleoylethanolamide. Lipids 43, 887-894.

9. Schwartz GJ, Fu J, Astarita G, et al. (2008) The lipid messenger OEA links dietary fat intake to satiety. Cell Metab $\mathbf{8}$, $281-288$

10. Terrazzino S, Berto F, Carbonare MD, et al. (2004) Stearoylethanolamide exerts anorexic effects in mice via down-regulation of liver stearoyl-coenzyme A desaturase-1 mRNA expression. FASEB J 18, 1580-1582.

11. Dalle Carbonare M, Del Giudice E, Stecca A, et al. (2008) A saturated $N$-acylethanolamine other than $N$-palmitoyl ethanolamine with anti-inflammatory properties: a neglected story. J Neuroendocrinol 20, 26-34.

12. Burstein S (2008) The elmiric acids: biologically active anandamide analogs. Neuropharmacology 55, 1259-1264.

13. Burstein SH \& Zurier RB (2009) Cannabinoids, endocannabinoids, and related analogs in inflammation. AAPS J 11, 109-119.

14. Calder PC (2007) Immunomodulation by omega-3 fatty acids. Prostaglandins Leukot Essent Fatty Acids 77, 327-335.

15. Calder PC (2008) The relationship between the fatty acid composition of immune cells and their function. Prostaglandins Leukot Essent Fatty Acids 79, 101-108.

16. Harris WS (2008) $n$-3 Fatty acids and health: DaVinci's code. Am J Clin Nutr 88, 595-596.

17. Harris WS \& Bulchandani D (2006) Why do omega-3 fatty acids lower serum triglycerides? Curr Opin Lipidol 17, 387-393.

18. Russo GL (2009) Dietary $n-6$ and $n-3$ polyunsaturated fatty acids: from biochemistry to clinical implications in cardiovascular prevention. Biochem Pharmacol 77, 937-946.

19. Artmann A, Petersen G, Hellgren LI, et al. (2008) Influence of dietary fatty acids on endocannabinoid and $\mathrm{N}$-acylethanolamine levels in rat brain, liver and small intestine. Biochim Biophys Acta 1781, 200-212.

20. Berger A, Crozier G, Bisogno T, et al. (2001) Anandamide and diet: inclusion of dietary arachidonate and docosahexaenoate leads to increased brain levels of the corresponding $\mathrm{N}$-acylethanolamines in piglets. Proc Natl Acad Sci U S A 98 , 6402-6406.

21. Bisogno T, Delton-Vandenbroucke I, Milone A, et al. (1999) Biosynthesis and inactivation of $\mathrm{N}$-arachidonoylethanolamine (anandamide) and $N$-docosahexaenoylethanolamide in bovine retina. Arch Biochem Biophys 370, 300-307.

22. Eckardt K, Sell H, Taube A, et al. (2009) Cannabinoid type 1 receptors in human skeletal muscle cells participate in the negative crosstalk between fat and muscle. Diabetologia 52, 664-674.

23. Balvers MG, Verhoeckx KC, Plastina P, et al. (2010) Docosahexaenoic acid and eicosapentaenoic acid are converted by 3T3-L1 adipocytes to $N$-acyl ethanolamines with anti-inflammatory properties. Biochim Biophys Acta 1801, 1107-1114.

24. Plastina P, Meijerink J, Vincken J-P, et al. (2009) Selective synthesis of unsaturated $N$-acylethanolamines by lipase-catalyzed $N$-acylation of ethanolamine with unsaturated fatty acids. Lett Org Chem 6, 444-447.

25. Lupinacci E, Meijerink J, Vincken JP, et al. (2009) Xanthohumol from hop (Humulus lupulus L.) is an efficient inhibitor of monocyte chemoattractant protein-1 and tumor necrosis factor-alpha release in LPS-stimulated RAW264.7 mouse macrophages and U937 human monocytes. J Agric Food Chem 57, 7274-7281.

26. Green LC, Wagner DA \& Glogowski J (1982) Analysis of nitrate, nitrite, and $\left[{ }^{15} \mathrm{~N}\right]$ nitrate in biological fluids. Anal Biochem 126, 131-138.

27. Calder PC (2009) Polyunsaturated fatty acids and inflammatory processes: new twists in an old tale. Biochimie $\mathbf{9 1}$, 791-795.

28. Svennerholm L (1968) Distribution and fatty acid composition of phosphoglycerides in normal human brain. J Lipid Res 9, 570-579.

29. Umhau JC, Zhou W, Carson RE, et al. (2009) Imaging incorporation of circulating docosahexaenoic acid into the human brain using positron emission tomography. J Lipid Res $\mathbf{5 0}$, $1259-1268$.

30. Wall KM, Diersen-Schade D \& Innis SM (1994) Plasma and tissue lipids of piglets fed formula containing saturated fatty acids from medium-chain triglycerides with or without fish oil. Am J Clin Nutr 59, 1317-1324.

31. Dallaire P, Bellmann K, Laplante M, et al. (2008) Obese mice lacking inducible nitric oxide synthase are sensitized to the metabolic actions of peroxisome proliferator-activated receptor-gamma agonism. Diabetes 57, 1999-2011.

32. Kuhlencordt PJ, Chen J, Han F, et al. (2001) Genetic deficiency of inducible nitric oxide synthase reduces atherosclerosis and lowers plasma lipid peroxides in apolipoprotein E-knockout mice. Circulation 103, 3099-3104.

33. Ponnuswamy P, Ostermeier E, Schrottle A, et al. (2009) Oxidative stress and compartment of gene expression determine proatherosclerotic effects of inducible nitric oxide synthase. Am J Pathol 174, 2400-2410.

34. Jobgen WS, Fried SK, Fu WJ, et al. (2006) Regulatory role for the arginine-nitric oxide pathway in metabolism of energy substrates. J Nutr Biochem 17, 571-588.

35. Bogdan C (2001) Nitric oxide and the immune response. Nat Immunol 2, 907-916.

36. Weldon SM, Mullen AC, Loscher CE, et al. (2007) Docosahexaenoic acid induces an anti-inflammatory profile in lipopolysaccharide-stimulated human THP-1 macrophages more effectively than eicosapentaenoic acid. $J$ Nutr Biochem 18, $250-258$.

37. Sun YX, Tsuboi K, Zhao LY, et al. (2005) Involvement of $N$-acylethanolamine-hydrolyzing acid amidase in the degradation of anandamide and other $\mathrm{N}$-acylethanolamines in macrophages. Biochim Biophys Acta 1736, 211-220.

38. Boring L, Gosling J, Cleary M, et al. (1998) Decreased lesion formation in CCR2 ${ }^{-/-}$mice reveals a role for chemokines in the initiation of atherosclerosis. Nature 394, 894-897.

39. Gu L, Okada Y, Clinton SK, et al. (1998) Absence of monocyte chemoattractant protein-1 reduces atherosclerosis in low density lipoprotein receptor-deficient mice. Mol Cell $\mathbf{2}$, 275-281.

40. Kanda H, Tateya S, Tamori Y, et al. (2006) MCP-1 contributes to macrophage infiltration into adipose tissue, insulin resistance, and hepatic steatosis in obesity. J Clin Invest 116, $1494-1505$.

41. Tateya S, Tamori Y, Kawaguchi T, et al. (2010) An increase in the circulating concentration of monocyte chemoattractant protein-1 elicits systemic insulin resistance irrespective of 
adipose tissue inflammation in mice. Endocrinology 151, 971-979.

42. Zhang M \& Fritsche KL (2004) Fatty acid-mediated inhibition of IL-12 production by murine macrophages is independent of PPARgamma. BrJ Nutr 91, 733-739.

43. Mickleborough TD, Tecklenburg SL, Montgomery GS, et al. (2009) Eicosapentaenoic acid is more effective than docosahexaenoic acid in inhibiting proinflammatory mediator production and transcription from LPS-induced human asthmatic alveolar macrophage cells. Clin Nutr 28, $71-77$.

44. Shi Y \& Pestka JJ (2009) Mechanisms for suppression of interleukin-6 expression in peritoneal macrophages from docosahexaenoic acid-fed mice. I Nutr Biochem 20, 358-368.

45. Schmitz G \& Ecker J (2008) The opposing effects of $n$-3 and n-6 fatty acids. Prog Lipid Res 47, 147-155.

46. Serhan CN, Chiang N \& Van Dyke TE (2008) Resolving inflammation: dual anti-inflammatory and pro-resolution lipid mediators. Nat Rev Immunol 8, 349-361.

47. Woodward DF, Carling RWC, Cornell CL, et al. (2008) The pharmacology and therapeutic relevance of endocannabinoid derived cyclo-oxygenase (COX)-2 products. Pharmacol Ther 120, 71-80.

48. White PJ \& Marette A (2006) Is omega-3 key to unlocking inflammation in obesity? Diabetologia 49, 1999-2001.

49. Watanabe S, Doshi M \& Hamazaki T (2003) n-3 Polyunsaturated fatty acid (PUFA) deficiency elevates and n-3 PUFA enrichment reduces brain 2-arachidonoylglycerol level in mice. Prostaglandins Leukot Essent Fatty Acids 69, 51-59.

50. Batetta B, Griinari M, Carta G, et al. (2009) Endocannabinoids may mediate the ability of $(n-3)$ fatty acids to reduce ectopic fat and inflammatory mediators in obese Zucker rats. J Nutr 139, 1495-1501.
51. Bisogno T, Melck D, De Petrocellis L, et al. (1998) Arachidonoylserotonin and other novel inhibitors of fatty acid amide hydrolase. Biochem Biophys Res Commun 248, $515-522$

52. Banni S \& Di Marzo V (2010) Effect of dietary fat on endocannabinoids and related mediators: consequences on energy homeostasis, inflammation and mood. Mol Nutr Food Res 54, 82-92.

53. Grundy SM (2006) Drug therapy of the metabolic syndrome: minimizing the emerging crisis in polypharmacy. Nat Rev Drug Discov 5, 295-309.

54. Hotamisligil GS (2006) Inflammation and metabolic disorders. Nature 444, 860-867.

55. Nathan C (2008) Epidemic inflammation: pondering obesity. Mol Med 14, 485-492.

56. Shoelson SE, Lee J \& Goldfine AB (2006) Inflammation and insulin resistance. J Clin Invest 116, 1793-1801.

57. Elizalde M, Rydén M, Van Harmelen V, et al. (2000) Expression of nitric oxide synthases in subcutaneous adipose tissue of nonobese and obese humans. J Lipid Res 41, 1244-1251.

58. Fujimoto M, Shimizu N, Kunii K, et al. (2005) A role for iNOS in fasting hyperglycemia and impaired insulin signaling in the liver of obese diabetic mice. Diabetes $\mathbf{5 4}$ $1340-1348$.

59. Engeli S, Janke J, Gorzelniak K, et al. (2004) Regulation of the nitric oxide system in human adipose tissue. J Lipid Res 45, 1640-1648.

60. Sugita H, Kaneki M, Tokunaga E, et al. (2002) Inducible nitric oxide synthase plays a role in LPS-induced hyperglycemia and insulin resistance. Am J Physiol Endocrinol Metab 282, E386-E394.

61. Philip CC (2008) Polyunsaturated fatty acids, inflammatory processes and inflammatory bowel diseases. Mol Nutr Food Res 52, 885-897. 